\title{
Generalized Assignment for Multi-Robot Systems via Distributed Branch-And-Price
}

\author{
Andrea Testa and Giuseppe Notarstefano
}

\begin{abstract}
C2021 IEEE. Personal use of this material is permitted. Permission from IEEE must be obtained for all other uses, in any current or future media, including reprinting/republishing this material for advertising or promotional purposes, creating new collective works, for resale or redistribution to servers or lists, or reuse of any copyrighted component of this work in other works.
\end{abstract}

\begin{abstract}
In this paper, we consider a network of agents that has to self-assign a set of tasks while respecting resource constraints. One possible formulation is the Generalized Assignment Problem, where the goal is to find a maximum payoff while satisfying capability constraints. We propose a purely distributed branch-and-price algorithm to solve this problem in a cooperative fashion. Inspired by classical (centralized) branchand-price schemes, in the proposed algorithm each agent locally solves small linear programs, generates columns by solving simple knapsack problems, and communicates to its neighbors a fixed number of basic columns. We prove finite-time convergence of the algorithm to an optimal solution of the problem. Then, we apply the proposed scheme to a generalized assignment scenario in which a team of robots has to serve a set of tasks. We implement the proposed algorithm in a ROS testbed and provide experiments for a team of heterogeneous robots solving the assignment problem.
\end{abstract}

\section{INTRODUCTION}

The Generalized Assignment Problem (GAP) is a well known combinatorial optimization problem with several applications as vehicle routing, facility location, resource scheduling and supply chain, to name a few [1], [2], [3]. Even though GAP is a NP-hard problem, several approaches have been developed for solving this problem both for exact and approximate solutions. We refer the reader to [4] for a survey. Branchand-price algorithms [2], [5] are among the most investigated algorithms allowing for both optimal and suboptimal solutions.

\section{A. Related Work}

Task assignment naturally arises in cooperative robotics, where heterogeneous agents collaborate to fulfill a complex task, see, e.g., [6] for an early reference. Specific applications include persistent monitoring of locations [7], path planning of mobile robots, e.g., UAVs [8], task scheduling for robots working in the same space [9], vehicle routing [10] and task assignment in urban environments [11]. All the previous problems are solved by means of centralized approaches.

This result is part of a project that has received funding from the European Research Council (ERC) under the European Union's Horizon 2020 research and innovation programme (grant agreement No 638992 - OPT4SMART).

A. Testa and G. Notarstefano are with the Department of Electrical, Electronic and Information Engineering, University of Bologna, Bologna, Italy, a.testa@unibo.it, giuseppe.notarstefano@unibo.it.
In order to deal with the computational complexity of the problem, a branch of literature analyzes parallel and decentralized approaches to the problem ${ }^{1}$ A well known parallel approach is the auction based one, originally proposed in [12]. A market-based approach is considered in [13] for the coordination of human-robot teams. Authors in [14] propose an algorithm, based on a sequential shortest augmenting path scheme, to solve a dynamic multi-task allocation problem. Agents propose assignments that are validated by a coordinating unit. As for decentralized schemes, authors in [15] solve a dynamic task allocation problem for robots that can perform local sensing operations and do not communicate among each other. In [16], a task assignment problem is solved, in a decentralized scheme, through the so called petal algorithm. In [17], a dynamic task assignment problem in which the cost vector changes in a bounded region is considered. A central unit is initially required but robots are able to exploit local communications to perform a reallocation if needed. An area partitioning problem for multi-robot systems is proposed in [18] and solved by a genetic algorithm. An area coverage problem in marine environments is solved in [19] with heuristics based on the traveling salesman problem.

As for distributed schemes, i.e., with processors in a peerto-peer network without a central coordinator, a distributed version of the Hungarian method is proposed in [20]. A distributed simplex scheme for degenerate linear programs (LP) is proposed in [21] in the context of multi-agent assignment problems. A distributed subgradient is applied in [22] to a task assignment problem, while in [23] a distributed column generation scheme is proposed. A linear task assignment problem with time-varying cost functions is considered in [24], while an optimal role and position assignment problem is addressed in [25] by iteratively solving a sequence of linear assignment problems. In these approaches, authors neglect integrality constraints on the decision variables, relying on the unimodular structure of the problems. As for distributed, suboptimal approaches for task assignment problems, in [26] a large-scale distributed task/target assignment problem across

\footnotetext{
${ }^{1}$ We denote parallel the approaches based on master-slave architectures, while we call decentralized the schemes with independent agents that do not communicate among each other.
} 
a fleet of autonomous UAVs is considered, but the communication graph is assumed to be complete. In the context of wireless sensor networks, [27] proposes a distributed task allocation in order to maximize the network life-time. A distributed task assignment algorithm is used in conjunction with a deterministic annealing in [28], in the context of limited-range sensor coverage. In [29], a dynamic vehicle routing problem is approached with a distributed protocol in which agents iteratively solve graph partitioning problems. In the works [30], [31] authors address Mixed-Integer Linear Programs by means of a distributed cutting-plane algorithm and apply it to a multi-agent multi-task assignment problem. Distributed implementations of the auction-based algorithm are often used to solve task assigment problems, see, e.g., [32] for an early reference, and in particular GAPs [33], [34]. The auction-based approach allows for a suboptimal solution with performance guarantees. In [35], this approach is applied to a task allocation problem expressed as a combinatorial optimization problem with matroid constraints. In the recent works [36], [37], a dynamic task allocation scenario with partial replanning is considered. The above references show that the exact resolution of GAP is an open problem in a purely distributed setting. Indeed, state-of-the-art solutions are usually based on proper linear relaxations or suboptimal approaches.

\section{B. Contributions}

In this paper, we propose a purely distributed version of the branch-and-price algorithm to solve the Generalized Assignment Problem by means of a network of agents. Specifically, each agent locally solves a linear programming relaxation of the GAP, generates columns by solving a (simple) knapsack problem, and exchanges estimates of the solution with neighboring agents. Due to the relaxation of the integrality constraints, the solution of this problem may not be feasible. Thus, new problems, based on the original one with suitable additional constraints, have to be solved. The set of these problems can be represented by a so called branching tree. By leveraging on their communication capabilities, agents explore their local trees until an optimal solution of the optimization problem has been found. With respect to the aforementioned works, and specifically [26], [32], [33], the proposed scheme has the following distinctive new features. To the best of the authors' knowledge, this is the first attempt to solve GAP to optimality in a purely distributed fashion. Remarkably, the proposed scheme is shown to converge also under time-varying and directed communication networks. Moreover, it is worth noticing that the approaches in [30], [31] are not applicable to the considered GAP scenario, which involves equality constraints. Finally, we apply the proposed algorithm to a dynamic task assignment problem where tasks may arrive during time and robots have to adapt the local plan according to the new information. An experimental platform, based on ROS (Robot Operating System), is proposed to run experiments in which a team of aerial and ground robots cooperatively solve the GAP relying on the proposed distributed branch-and-price scheme.

The paper unfolds as follows. In Section II we introduce the distributed setup considered throughout the paper. Then, we introduce the Generalized Assignment Problem and a centralized scheme, called branch-and-price, to solve it. In Section III we propose a purely distributed branch-and-price algorithm. In Section IV we provide numerical simulations for randomly generated GAPs and in Section $\mathrm{V}$ we show the results of experiments on a swarm of heterogeneous robots.

Notation: We denote by $e_{\ell}$ the $\ell$-th vector of the canonical basis (e.g., $e_{1}=\left[\begin{array}{llll}1 & 0 \ldots 0\end{array}\right]^{\top}$ ) of proper dimension. Given a vector $v_{\ell} \in \mathbb{R}^{d}$, we denote by $v_{\ell_{m}}$ the $m$-th component of $v_{\ell}$. Also, we denote by $1_{r}\left(0_{r}\right)$ the vector in $\mathbb{R}^{r}$ with all its entries equal to $1(0)$.

\section{Distributed Setup AND PREliminaries}

In this section, we introduce the distributed setup for the Generalized Assignment Problem addressed in the paper. Also, the (centralized) branch-and-price scheme is illustrated.

\section{A. Distributed Problem Setup}

In the Generalized Assignment Problem, the objective is to find a maximal profit assignment of $M$ tasks to $N$ agents such that each task is assigned only to one agent. In this scenario, the generic agent $i$ has a reward $p_{i m} \in \mathbb{R}$ if it executes the $m$-th task. It also has a limited capacity $g_{i} \in \mathbb{R}$ and it uses an amount $w_{i m} \in \mathbb{R}$ of capacity if it performs the $m$-th task. Let $x_{i m}$ be a binary variable indicating whether task $m$ is assigned to agent $i\left(x_{i m}=1\right)$ or not $\left(x_{i m}=0\right)$. We denote constraints in the form $x_{i m} \in\{0,1\}$ as integer constraint. Then, the standard integer programming formulation is

$$
\begin{aligned}
\underset{x_{11}, \ldots, x_{N M}}{\max } & \sum_{i=1}^{N} \sum_{m=1}^{M} p_{i m} x_{i m} \\
\text { subj. to } & \sum_{i=1}^{N} x_{i m}=1, m=1, \ldots, M, \\
& \sum_{m=1}^{M} w_{i m} x_{i m} \leq g_{i}, i=1, \ldots, N, \\
& x_{i m} \in\{0,1\}, i=1, \ldots, N, m=1, \ldots, M .
\end{aligned}
$$

In order to streamline the notation, we now introduce a formulation of the GAP better highlighting the structure of the problem in a distributed scenario. Let $z_{i}=\left[x_{i 1}, \ldots, x_{i M}\right]^{\top} \in$ $\mathbb{R}^{M}, \forall i=1, \ldots, N$. In the following we denote as $z$ the stack $\left[z_{1}^{\top}, \ldots, z_{N}^{\top}\right]^{\top}$. Also, let $c_{i}=\left[p_{i 1}, \ldots, p_{i M}\right]^{\top} \in \mathbb{R}^{M}, D_{i}=$ $\left[w_{i 1}, \ldots, w_{i M}\right] \in \mathbb{R}^{M}$ and $P_{i}=\left\{z_{i} \in\{0,1\}^{M} \mid D_{i} z_{i} \leq g_{i}\right\}$, for $i=1, \ldots, N$. Then, (1) can be recast as

$$
\begin{array}{ll}
\underset{z_{1}, \ldots, z_{N}}{\max } & \sum_{i=1}^{N} c_{i}^{\top} z_{i} \\
\text { subj. to } & \sum_{i=1}^{N} z_{i}=1_{M}, \\
& z_{i} \in P_{i}, i=1, \ldots, N .
\end{array}
$$

This new formulation of (1) allows us to point out the distributed nature of the problem. Namely, $c_{i}$ describes the profits associated to assigning tasks to agent $i, \sum_{i=1}^{N} z_{i}=1_{M}$ describes the assignment constraints (coupling constraints), 
$P_{i}$ describes the capacity restrictions on the agents (local constraints). It is worth noting that the sets $P_{i}$ are bounded for $i=1, \ldots, N$.

The agents must solve (2) cooperatively in a distributed fashion with limited communication and computation capabilities, as well as limited memory. We consider the natural scenario in which the $i$-th agent only knows the polyhedron $P_{i}$ and the cost vector $c_{i}$, thus not having knowledge of other agent data. In order to solve the problem, agents can exchange information according to a time-varying communication network modeled as a time-varying digraph $\mathcal{G}^{t}=$ $\left(\{1, \ldots, N\}, \mathcal{E}^{t}\right)$, with $t \in \mathbb{N}$ being a universal slotted time representing a temporal information on the graph evolution. Notice that time $t$ does not need to be known by the agents. A digraph $\mathcal{G}^{t}$ models the communication in the sense that there is an edge $(i, j) \in \mathcal{E}^{t}$ if and only if agent $i$ is able to send information to agent $j$ at time $t$. For each node $i$, the set of in-neighbors of $i$ at time $t$ is denoted by $\mathcal{N}_{i, t}^{\text {in }}$ and is the set of $j$ such that there exists an edge $(j, i) \in \mathcal{E}^{t}$. A static digraph is said to be strongly connected if there exists a directed path for each pair of agents $i$ and $j$. Next, we require the following.

Assumption 2.1 (Graph Connectivity): The communication graph is $L$-strongly connected, i.e., there exists an integer $L \geq$ 1 such that, for all $t \in \mathbb{N}$, the graph $\left(\{1, \ldots, N\}, \bigcup_{\tau=t}^{t+L-1} \mathcal{E}^{\tau}\right)$ is strongly connected.

Notice that this is a standard, mild, assumption in the context of distributed optimization that allows to model direct, timevarying, asynchronous and possibly unreliable communication.

\section{B. Centralized Branch-and-Price Method}

We now introduce the main concepts regarding the branchand-price scheme. We refer the reader to [5] for a more detailed dissertation. For the sake of clarity, we organize this subsection in three parts.

\section{Dantzig-Wolfe Decomposition for GAPs}

An equivalent formulation of (2), which is exploited in our distributed setup, can be obtained as follows. Such procedure, originally introduced in [5], is strictly related to the DantzigWolfe Decomposition [38]. Points $z_{i} \in P_{i}$ can be represented as the linear combination of a finite number of vectors $v_{i}^{q}$, with $q \in\left\{1, \ldots,\left|Q_{i}\right|\right\}$, i.e.,

$$
z_{i}=\sum_{q=1}^{\left|Q_{i}\right|} v_{i}^{q} \lambda_{i}^{q},
$$

where we denote with $Q_{i}$ the set of vectors $v_{i}^{q}$. The variables $\lambda_{i}^{q}, \forall q=1, \ldots,\left|Q_{i}\right|$, also called combiners, have to satisfy

$$
\begin{aligned}
& \sum_{q=1}^{\left|Q_{i}\right|} \lambda_{i}^{q}=1, \\
& \lambda_{i}^{q} \in\{0,1\} .
\end{aligned}
$$

It can be shown that, for GAPs, $Q_{i}$ coincides with the set of extreme points of the convex hull $\operatorname{conv}\left(P_{i}\right)$ of $P_{i}$, [5]. Let $\Lambda \in \mathbb{R}^{\sum_{i=1}^{N}\left|Q_{i}\right|}$, be the stack of all the combiners.

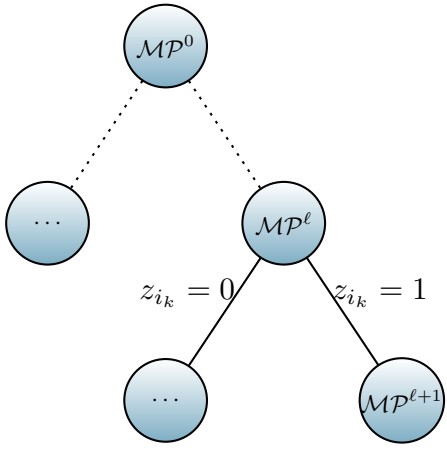

Fig. 1. Example of branching tree with problems generated according to constraints on the sets $P_{i}$.

Substituting (3) and (4) in (2) leads to the following equivalent Integer Programming Master Problem (IP-MP)

$$
\begin{aligned}
\max _{\Lambda} & \sum_{i=1}^{N} \sum_{q=1}^{\left|Q_{i}\right|}\left(c_{i}^{\top} v_{i}^{q}\right) \lambda_{i}^{q} \\
\text { subj. to } & \sum_{i=1}^{N} \sum_{q=1}^{\left|Q_{i}\right|} v_{i}^{q} \lambda_{i}^{q}=1_{M}, \\
& \sum_{q=1}^{\left|Q_{i}\right|} \lambda_{i}^{q}=1, i=1, \ldots, N, \\
& \lambda_{i}^{q} \in\{0,1\}, q \in\left\{1, \ldots,\left|Q_{i}\right|\right\}, i=1, \ldots, N .
\end{aligned}
$$

Notice that an optimal solution $z^{\star}$ of (2) can be retrieved from an optimal solution $\Lambda^{\star}$ of (5) by substituting the entries of $\Lambda^{\star}$ in [3], [2].

\section{Branching Tree}

The presence of binary constraints makes $(5)$ hard to solve. In order to find an optimal solution to the problem, the branchand-price algorithm, [5], explores the set of feasible solutions of GAP by iteratively generating and solving relaxed versions of (5) including suitable, tightening constraints. That is, the constraint $\lambda_{i}^{q} \in\{0,1\}$ of all these problems is relaxed to $\lambda_{i}^{q} \geq 0\left(\lambda_{i}^{q} \leq 1\right.$ can be omitted as it is implicit in the constraint $\left.\sum_{q=1}^{\left|\bar{Q}_{i}\right|} \lambda_{i}^{q}=1\right)$. These problems can be represented as nodes of a so called branching tree, see, e.g., Figure 1 The $\ell$-th node of the tree to be solved represents a problem, in the form of (5), obtained by relaxing the integer constraints and enforcing the constraints of the edges. We denote such problem as $\mathcal{M} \mathcal{P}^{\ell}$, by $\Lambda^{\star \ell}$ its optimal solution and by $z^{\star \ell}$ and $J^{\star \ell}$ the solution and cost in terms of the variables $z$. Branches (edges) indicate the constraints that have to be added to generate new problems. Instead of considering constraints on the $\Lambda$ variables, new problems are generated by including, to the sets $P_{i}$, additional constraints in the form $z_{i_{k}}=0, z_{i_{k}}=1$ for some $z_{i_{k}}(\ell) \notin\{0,1\}$. In the following, we assume there exists an extraction strategy to determine the next node of the tree to be solved and a rule to choose the index $i_{k}$. Let $P_{i}^{\ell}$ be the sets obtained by including such additional constraints and let $Q_{i}^{\ell}$ be the sets of extreme points of $\operatorname{conv}\left(P_{i}^{\ell}\right)$. Since $Q_{i}^{\ell} \subset Q_{i}$, this results in generating relaxed problems in the form of (5) 
with less optimization variables. The algorithm keeps track of a lowerbound, also called incumbent, $J^{\mathrm{INC}}$ on the cost, and of a candidate solution $z^{\mathrm{INC}}$. After solving the generic problem $\mathcal{M P}^{\ell}$, one of the following operations is performed:

- Incumbent Update: If $z^{\star \ell} \in\{0,1\}^{N M}$ and $J^{\star \ell} \geq J^{\mathrm{INC}}$, then $J^{\mathrm{INC}}=J^{\star \ell}$ and $z^{\mathrm{INC}}=z^{\star \ell}$.

- Branching: If $J^{\star \ell}>J^{\mathrm{INC}}$ and $z^{\star \ell} \notin\{0,1\}^{N M}$ and $J^{\star \ell} \geq$ $J^{\mathrm{INC}}$, two new problems are added to the tree.

- Pruning: If $J^{\star \ell} \leq J^{\mathrm{INC}}$ or $\mathcal{M P} \mathcal{P}^{\ell}$ is infeasible, nothing is done.

Remark 2.2: Pruning prevents the algorithm from inspecting problems that do not improve $J^{\mathrm{INC}}$.

At the end of the algorithm $J^{\mathrm{INC}}$ and $z^{\mathrm{INC}}$ coincide with the optimal cost $J^{\star}$ and solution $z^{\star}$ of (2).

\section{Column Generation}

Each problem $\mathcal{M P}^{\ell}$ has a large number of optimization variables. Thus, it can be approached by means of the so called column generation algorithm (originally proposed in [39] in the context of cutting stock problems). It consists in iteratively performing the following three steps:

i) A Restricted Master Problem (RMP) is solved, made by a small subset of the columns $s^{2}$ of $\mathcal{M} \mathcal{P}^{\ell}$ with sets $\bar{Q}_{i}^{\ell} \subset Q_{i}^{\ell}$ and a smaller combiner vector $\Lambda$, i.e.,

$$
\begin{aligned}
\max _{\tilde{\Lambda}} & \sum_{i=1}^{N} \sum_{q=1}^{\left|\bar{Q}_{i}^{\ell}\right|}\left(c_{i}^{\top} v_{i}^{q}\right) \tilde{\lambda}_{i}^{q} \\
\text { subj. to } & \sum_{i=1}^{N} \sum_{q=1}^{\left|\bar{Q}_{i}\right|} v_{i}^{q} \tilde{\lambda}_{i}^{q}=1_{M}, \\
& \sum_{q=1}^{\left|\bar{Q}_{i}^{\ell}\right|} \tilde{\lambda}_{i}^{q}=1, i=1, \ldots, N, \\
& \tilde{\lambda}_{i}^{q} \geq 0, q \in\left\{1, \ldots,\left|\bar{Q}_{i}^{\ell}\right|\right\}, i=1, \ldots, N .
\end{aligned}
$$

ii) If possible, new columns are added to the RMP in order to improve the current solution. Let $\left[\pi^{\top} \mu^{\top}\right]^{\top}$ be a dual optimal solution of (6) at a generic iteration of the algorithm. In particular, $\pi \in \mathbb{R}^{M}$ is associated to the constraint $\sum_{i=1}^{N} \sum_{q \in \bar{Q}_{i}^{\ell}} v_{i}^{q} \tilde{\lambda}_{i}^{q}=1_{M}$, while $\mu \in \mathbb{R}^{N}$ is associated to the constraint $\sum_{q \in \bar{Q}_{i}^{e}} \tilde{\lambda}_{i}^{q}=1$. For each $i=1, \ldots, N$ a new column is found by solving the so called pricing problem:

$$
\begin{gathered}
\bar{v}_{i} \in \underset{z_{i}}{\operatorname{argmax}}\left(c_{i}-\pi\right)^{\top} z_{i} \\
\text { subj. to } z_{i} \in P_{i}^{\ell} .
\end{gathered}
$$

Consider now the associated column in the form $h_{i}=$ $\left[\begin{array}{lll}c_{i}^{\top} \bar{v}_{i} & \bar{v}_{i}^{\top} & e_{i}^{\top}\end{array}\right]^{\top}$. Then, $h_{i}$ allows for a cost improvement if it has positive reduced cost, i.e., if $\left(c_{i}-\pi\right) \bar{v}_{i}-\mu_{i}>0$.

iii) A pivoting operation is performed, that is, all columns with positive reduced cost are included in the RMP, while columns of the RMP that are not associated to basic variables are dropped ${ }^{3}$ Then, the procedure is iterated until no more

\footnotetext{
${ }^{2}$ We refer the reader to Appendix A for the definition of column for a linear program. Informally, a column is a portion of the cost and constraint vectors associated to a decision variable.

${ }^{3}$ We refer the reader to Appedix A for the definition of basic variables.
}

TABLE I

LIST OF THE MAIN SYMBOLS AND THEIR DEFINITIONS

\begin{tabular}{ll}
\hline & Standard GAP Formulation \\
\hline$N \in \mathbb{N}_{>0}$ & Number of robots \\
$M \in \mathbb{N}_{>0}$ & Number of tasks \\
$x_{i m} \in\{0,1\}$ & 1 if robot $i$ serves task, 0 otherwise \\
$z_{i} \in \mathbb{R}^{M}$ & {$\left[x_{i 1}, \ldots, x_{i M}\right]^{\top}$} \\
$p_{i m} \in \mathbb{R}_{\geq 0}$ & Reward if robot $i$ serves task $m$ \\
$c_{i} \in \mathbb{R}^{M}$ & {$\left[p_{i 1}, \ldots, p_{i M}\right]^{\top}$} \\
$g_{i} \in \mathbb{R}_{\geq 0}$ & Capacity of robot $i$ \\
$w_{i m} \in \mathbb{R}_{\geq 0}$ & Capacity consumption of task $m$ for robot $i$ \\
$D_{i} \in \mathbb{R}^{M}$ & {$\left[w_{i 1}, \ldots, w_{i M}\right]$} \\
$P_{i} \subseteq\{0,1\}^{M}$ & $\left\{z_{i} \in\{0,1\}^{M} \mid D_{i} z_{i} \leq g_{i}\right\}$ \\
\hline \multicolumn{3}{c}{ Dantzig-Wolfe Reformulation } \\
\hline$Q_{i}$ & set of extreme points of $P_{i}$ \\
$v_{i}^{q} \in\{0,1\}^{M}$ & $q$-th extreme point of $P_{i}$ \\
$\lambda_{i}^{q} \in\{0,1\}$ & Combiner associated to $v_{i}^{q}$ \\
$\Lambda \in \mathbb{R}^{\sum_{i=1}^{N}\left|Q_{i}\right|}$ & Stack of combiners \\
\hline \multicolumn{2}{c}{ Branch-and-Price } \\
\hline $\mathcal{M P} \mathcal{P}^{\ell}$ & $\quad$-th node of the branching tree \\
$z^{\star \ell}, J^{\star \ell}$ & Optimal solution and cost of $\mathcal{M P} \mathcal{P}^{\ell}$ \\
$P_{i}^{\ell}$ & Constraint set of robot $i$ at node $\mathcal{M P} \mathcal{P}^{\ell}$ \\
$z^{\mathrm{INC}}, J^{\mathrm{INC}}=J^{\star \ell}$ & Candidate GAP solution and cost \\
\hline &
\end{tabular}

columns with positive reduced cost can be found. Let $\Lambda^{\star \ell}$ be the final optimal solution of the relaxed version of (5) obtained with this procedure. Let $\bar{\lambda}_{i}^{q}$ be the entry of $\Lambda^{\star \ell}$ associated to a vertex $v_{i}^{q} \in Q_{i}^{\ell}$. Then, the solution $z^{\star \ell}$ of $\mathcal{M P}^{\ell}$ can be expressed as (c.f. [3])

$$
z_{i}(\ell)=\sum_{q=1}^{\left|Q_{i}^{\ell}\right|} \bar{\lambda}_{i}^{q} v_{i}^{q}, \quad i=1, \ldots, N .
$$

Remark 2.3: When applying the Dantzig-Wolfe Decomposition, we follow the approach in [5] and do not relax the binary constraints in (2). This results in local knapsack problems (7) that can be efficiently solved through dynamic programming schemes, [40].

We collect in Table 1 all the relevant symbols.

\section{Distributed Branch-And-Price Method}

In this section, we provide a purely distributed algorithm, inspired by the centralized branch-and-price scheme, to solve (2) in a peer-to-peer network. We assume a solver for Linear Programs is available. In particular, we use the simplex algorithm proposed in [41] to find the unique lexicographically minimal optimal solution of a LP and the associated optimal basis.

In the proposed distributed algorithm, called Distributed Branch-and-Price, each agent $i$ maintains and updates, at the generic time $t$, local optimal cost and solution candidates $J_{i}^{t}$ and $z_{[i]}^{t}$, as well as a local tree $\mathcal{T}_{i}^{t}$. Each agent also maintains and updates a label $\mathcal{L}_{i}^{t}$ indicating which problem in $\mathcal{T}_{i}^{t}$ it is solving. The candidate optimal solution of a generic problem $\mathcal{M} \mathcal{P}_{i}^{\ell}$ of $\mathcal{T}_{i}^{t}$, for some $\ell$, is characterized in terms of a small, representative set of columns called basis (c.f. Appendix A). 
We denote as $B_{i}^{t}$ the candidate optimal basis of agent $i$ at time $t$. At each communication round $t$, the generic agent $i$ constructs a local restricted master program $\mathrm{RMP}_{i}$ in the form

$$
\begin{aligned}
\max _{\tilde{\Lambda}_{i}} & \bar{c}_{V, i}^{\top} \tilde{\Lambda}_{i} \\
\text { subj. to } & \bar{V}_{i} \tilde{\Lambda}_{i}=1_{M}, \\
& \left(1_{N} 1_{\left|\tilde{\Lambda}_{i}\right|}^{\top}\right) \tilde{\Lambda}_{i}=1_{N}, \\
& \tilde{\Lambda}_{i} \geq 0_{\left|\tilde{\Lambda}_{i}\right|} .
\end{aligned}
$$

Notice that this problem has the same structure as (6) where the columns are the ones of the bases $B_{j}^{t}$ with $j \in \mathcal{N}_{i, t}^{\text {in }}$. To streamline the notation, we denote by $\bar{V}_{i}$ the stack of vertexes $v_{i}^{q}$ received by the agent, by $\bar{c}_{V, i}$ the stack of related costs $c_{i}^{\top} v_{i}^{q}$ and by $\tilde{\Lambda}_{i}$ the optimization variable. Agent $i$ solves its local $\mathrm{RMP}_{i}$, updates the candidate basis $B_{i}^{t}$, and recovers the associated optimal dual variables $\left[\begin{array}{ll}\pi_{i}^{t} & \mu_{i}^{t}\end{array}\right]$. With the dual solution of the local $\mathrm{RMP}_{i}$ at hands, agent $i$ solves a pricing problem

$$
\begin{aligned}
& \max _{z_{i}}\left(c_{i}-\pi_{i}^{t}\right)^{\top} z_{i} \\
& \text { subj. to } z_{i} \in P_{i}^{t},
\end{aligned}
$$

which has the same structure as (7). As discussed in Section $\Pi$. this allows agents to generate a new column $h_{i}{ }_{4}^{4}$ If such column improves the overall cost, i.e., it has positive reduced cost, agent $i$ substitutes one column of $B_{i}^{t}$ with $h_{i}$. This is done according to a so called PIVOT operation.

Each time an agent detects convergence, or receives a label $\mathcal{L}_{j}^{t}>\mathcal{L}_{i}^{t}$ from some neighbor $j \in \mathcal{N}_{i, t}^{\text {in }}$, it sets $\mathcal{L}_{i}^{t+1}=\mathcal{L}_{i}^{t}+1$. Then, it retrieves the local cost and solution $J_{i}^{\mathrm{LP}}, z_{[i]}^{\mathrm{LP}}$ from $B_{i}^{t+1}$ through a EXTRACTSOL function. If $J_{i}^{\mathrm{LP}} \geq J_{i}^{t}$ and $z_{[i]}^{\mathrm{LP}} \in\{0,1\}^{N M}$, it updates the local candidate optimal cost and solution as $J_{i}^{t+1}=J_{i}^{\mathrm{LP}}, z_{[i]}^{t+1}=z_{[i]}^{\mathrm{LP}}$. Otherwise, it sets $J_{i}^{t+1}=J_{i}^{t}, z_{[i]}^{t+1}=z_{[i]}^{t}$. If $J_{i}^{\mathrm{LP}} \geq J_{i}^{t}$ but $z_{[i]}^{\mathrm{LP}} \notin\{0,1\}^{N M}$ it performs a branching operation. We denote by BRANCH the routine that updates $\mathcal{T}_{i}^{t}$ according to a branching on $z_{[i]}^{\mathrm{LP}}$. Finally, the agent starts to solve a new problem, if any, by updating, through an EXTRACTCONSTR function, the local constraint set $P_{i}^{t+1}$. From now on we assume that the routines BRANCH and EXTRACTCONSTR are common to all the agents. The whole procedure is summarized in Table 1 from the perspective of agent $i$.

The convergence properties of the Distributed Branch-andPrice algorithm are stated in the next theorem.

Theorem 3.1: Let (2) be feasible and Assumption 2.1 hold. Consider the sequences $\left\{J_{i}^{t}, z_{[i]}^{t}\right\}_{t \geq 0}, i \in\{1, \ldots, N\}$ generated by the Distributed Branch-and-Price algorithm. Then, in a finite number $\bar{T} \in \mathbb{N}$ of communication rounds, agents agree on a common optimal solution $z^{\star}$ with optimal cost value $J^{\star}$ of (2), i.e., $J_{i}^{t}=J^{\star}$ and $z_{[i]}^{t}=z^{\star}, \forall i \in\{1, \ldots, N\}$ and $\forall t \geq \bar{T}$.

We refer the reader to Appendix $B$ for the proof of Theorem 3.1

\footnotetext{
${ }^{4}$ Here, $P_{i}^{t}=\left\{z_{i} \in\{0,1\}^{M} \mid z_{i} \in P_{i}, z_{i} \in \Delta_{i}^{\ell}\right\}$, with $\Delta_{i}^{\ell}$ being the set of branching binary constraints associated to the problem $\mathcal{M} \mathcal{P}_{i}^{\ell}$ that agent $i$ is solving at iteration $t$.
}

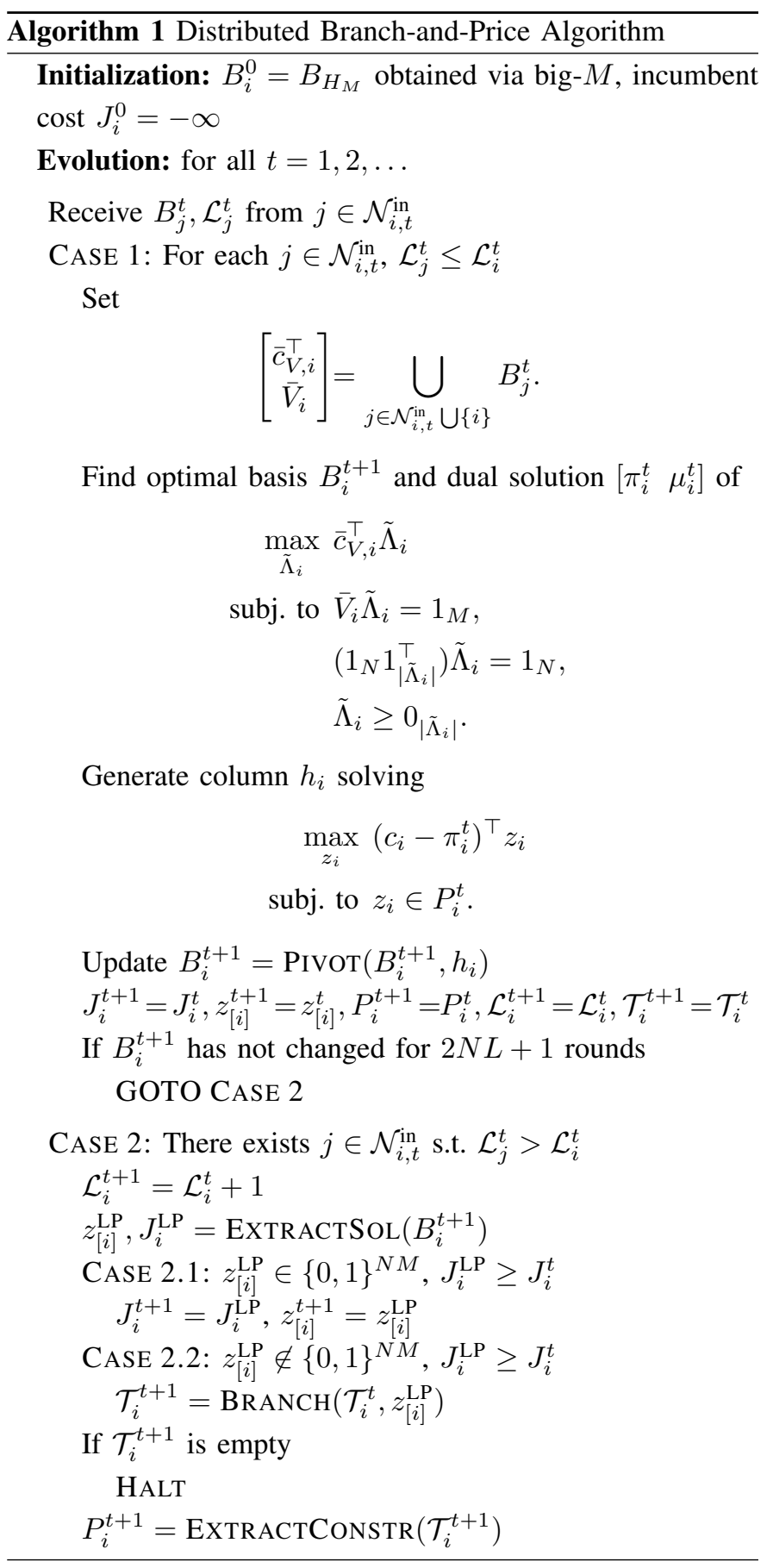

We discuss some interesting features of the proposed distributed scheme. First, agents do not need to know the universal slotted time $t$. That is, agents can run the steps of the distributed algorithm according to their own local clock. If an agent is performing its computation it is assumed not to have outgoing edges on the communication graph and the steps are performed accordingly to the available in-neighbor bases. This implies that the proposed distributed scheme works under asynchronous communication networks. Second, as it will be shown in the analysis, the $i$-th agent can detect that convergence to an optimal basis has occurred if its basis $B_{i}^{t}$ does not change for $2 L N+1$ communication rounds. In this way, it can halt the steps in CASE 1 of Algorithm 1 
Third, during the first iterations an agent $i$ may not have enough information to solve the $\mathrm{RMP}_{i}$ (9). Thus, it plugs into the local problem a set of artificial variables, eventually discarded during the evolution of the algorithm, with high cost. This method, also called Big-M method, allows the agents to always find a solution to the $\mathrm{RMP}_{i}$. As for the communication overhead, at each communication round each robot sends to its neighbors a matrix of size $(N+M+1) \times(N+M)$. Each column of this matrix is in the form $h_{i}=\left[\begin{array}{lll}c_{i}^{\top} \bar{v}_{i} & \bar{v}_{i}^{\top} & e_{i}^{\top}\end{array}\right]^{\top}$. Here, $c_{i}^{\top} \bar{v}_{i}$ is a real number specifying the cost to execute an allocation $\bar{v}_{i} \in\{0,1\}^{M}$. The vector $e_{i}^{\top} \in\{0,1\}^{N}$ specifies which robot generated that allocation. It is worth noting that the vector $\bar{v}_{i}$ can be encoded as an array of $M$ bits while $e_{i}^{\top}$ can be encoded as an integer number. Finally, we underline that the assumption that (2) is feasible can be relaxed to include unfeasible GAPs, but this assumption allows us to lighten the discussion.

Remark 3.2: As a possible variation, agents may harness the communication with a Cloud node to speed-up the convergence time, and reduce the local memory and computing requirements. In this architecture the cloud unity is only involved in the storage of the branching tree (and not in the column generation steps). Thereby, agents do not construct local branching trees. Also, agent data remain private and the number of messages exchanged at each communication round does not increase. When an agent $i$, at time $\bar{t}_{\ell}$, detects that convergence to an optimal solution of a problem $\mathcal{M P}^{\ell}$ has occurred, it sends the basis $B_{i}^{\bar{t}_{\ell}}$ to the Cloud. At this point, the cloud extracts the optimal cost and solution $J^{\star \ell}$ and $z^{\star \ell}$ and analyzes them according to the steps in CASE 2 of Algorithm 11 Finally, if the tree is not empty, it extracts a new problem $\mathcal{M P}^{\ell+1}$ from the tree according to the extraction strategy, and broadcasts to each agent $i$ the additional constraints to build-up $P_{i}^{\ell+1}$. The proof of the cloud-based version follows similar arguments as the one of Theorem 3.1 and is omitted.

\section{NUMERICAL COMPUTATIONS}

In order to assess the performance and highlight the main features of our distributed algorithm, we provide a set of numerical computations. Simulations have been implemented on the DISROPT [42] toolbox and carried out on a laptop equipped with a $2.5 \mathrm{GHz}$ dual core processor and $16 \mathrm{~GB}$ of RAM. In the following, we generate new problems, during the branching procedure, by adding constraints in the form $z_{i_{k}}=0$ and $z_{i_{k}}=1$, where $z_{i_{k}}$ is the first non-integer entry of the vector $z$. Regarding the order in which problems are extracted and solved, we adopt the widely used depth first selection procedure, [40]. In this approach, the generated problems are stored in a stack, thereby the extraction procedure follows a LIFO approach. Each time a branching occurs, the new problems are placed on the top of the stack. In our implementation, we insert in the first position the problem in which $z_{i_{k}}=0$ is added at last.

We perform Monte Carlo simulations on random GAP instances. We generate such instances according to four different random models, usually referred to as Model A, B, C and
$\mathrm{D}$, of increasing difficulty. We refer the reader to [2] for a survey on such models. Let $\mathcal{U}(a, b)$ denote the discrete uniform distribution on the interval $[a, b]$. The data are generated as follows.

- Model A: $w_{\ell m}^{A} \in \mathcal{U}(10,25), p_{\ell m}^{A} \in \mathcal{U}(5,25)$ and $g_{\ell}^{A}=9(M / N)+0.4 \max _{1 \leq \ell \leq N} \sum_{m \in \mathcal{J}_{\ell}^{\star}} w_{\ell m}$, with $\mathcal{J}_{\ell}^{\star}:=\left\{m \mid \ell=\operatorname{argmin}_{r} p_{r m}\right\}$.

- Model B: $w_{\ell m}^{B}=w_{\ell m}^{A}, p_{\ell m}^{B}=p_{\ell m}^{A}$ and $g_{\ell}^{B}=0.7 g_{\ell}^{A}$.

- Model C: $w_{\ell m}^{C}=w_{\ell m}^{A}, p_{\ell m}^{C}=p_{\ell m}^{A}$ and $g_{\ell}^{C}=$ $\sum_{1 \leq m \leq M} w_{\ell m} / m$.

- Model D: $w_{\ell m}^{D} \in \mathcal{U}(1,100), p_{\ell m}^{D}=100-w_{\ell m}+k$, with $k \in \mathcal{U}(1,21)$ and $g_{\ell}^{D}=g_{\ell}^{C}$.

We consider different scenarios by varying the number of agents and tasks, thus considering problems with different size and task-over-agents ratio. As for the number of agents, $N=$ $5,10,15$, while, for the number of tasks, $M=20,30$.

We generate 50 random instances for each scenario and for each model. We are interested in both time and memory performance of the distributed algorithm. Thus, we show the time that is needed to terminate the algorithm, expressed in terms of the number of communication rounds, and the maximum number of tree nodes stored by the agents. We also show the equivalent time, in seconds, needed for each simulation. Since DISROPT exploits the MPI protocol to simulate the agents, the computation time per-agent is evaluated as $T_{a g}=T_{e l} N_{c o} / N$ where $T_{e l}$ is the total elapsed time and $N_{c o}$ is the number of cores. As the problem size increases, the solution of these problems requires the exploration of thousands of tree nodes, see, e.g., [2]. However, in practical scenarios where assignment problems have to be solved almost in realtime, it is useful to consider a feasible sub-optimal solution to the problem instead of an optimal one. Thereby, even though our algorithm is able to find an optimal solution, in the proposed simulations agents interrupt the distributed algorithm when they find a feasible (sub-optimal) solution. For this reason, we also provide the relative error, in terms of cost value, between the exact solution (evaluated through a centralized solver) and the solution found by the agents. As for the connectivity among agents, we consider a static network modeled by a cyclic digraph. We underline that our algorithm adapts to more complex graph models. However, the choice of such digraph is interesting for simulation purposes due to the fact that it is the static digraph with largest diameter. Thus, the expected number of communication rounds to completion is expected to be higher with respect to graphs with smaller diameter.

The mean value and the standard deviation (evaluated over the number of trials) for each simulation scenario are shown in Table II We highlight that, in all the simulations, the average relative error is always below $5 \%$. The time to convergence increases with the task-to-agent ratio $(M / N)$. As an example, see Table II Model A with $N=15$ and $M=30$ requires less communication rounds than Model A with $N=5$ and $M=30$, even though the overall number of optimization variable is larger. This behavior of the Distributed Branchand-Price algorithm appears to be consistent with the one reported in the literature for centralized methods. Similarly, Model D is far more difficult to be solved than Model 


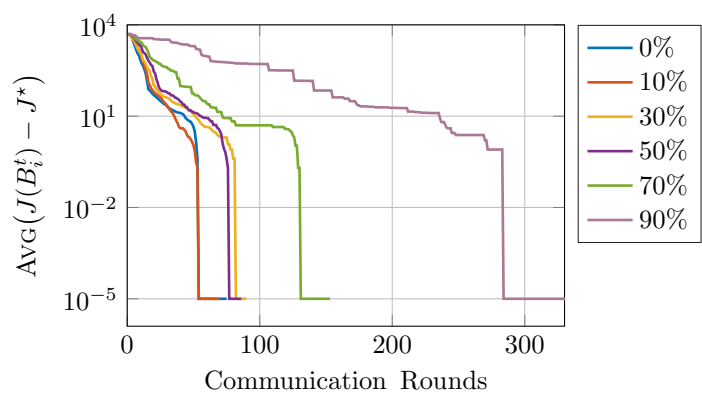

Fig. 2. Cost error during the evolution of the algorithm with different percentages of packet loss.

A and requires more communication rounds (see, e.g, the communication rounds needed to solve Model A and Model D with $N=5, M=20$ ). We underline that the number of communication rounds strictly depends on the graph diameter. Since we run the algorithm on a cyclic digraph, whose diameter is $N-1$, the results provided in Table $\Pi$ II are the ones expected in case of loose connectivity. The maximum number of stored nodes exhibits a similar behavior. That is, as the task-to-agent ratio increases and more difficult models are considered, the distributed algorithm has to explore more branches. To conclude, we propose a numerical simulation in which robots communicate in a network subject to packet loss. We consider a scenario with $N=5$ and $M=20$. Problem data are generated according to Model A. We consider the cases with loss probability $0 \%$ (no packet loss), $10 \%, 30 \%, 50 \%, 70 \%, 90 \%$. Specifically, at each iteration, the $i$-th robot discards the message from the $j$-th robot according to the given probability. Results are given in Figure 2 . We show the mean error between the cost $J\left(B_{i}^{t}\right)$ associated to the basis $B_{i}^{t}$ and the optimal solution $J^{\star}$.

Remark 4.1: Other distributed approaches suitable for the GAP solution are the ones in [32], [33]. As for the one in [32], authors consider the case in which $w_{i m} \in\{0,1\}$ for each $i \in\{1, \ldots, N\}$ and for each $m \in\{1, \ldots, M\}$ (cf. (1)). When the cost function is linear, as in the GAP scenario, the constraint matrix is said to be totally unimodular and the problem can be solved as a linear problem instead of a mixed-integer problem. Thereby, the first solution found by our algorithm, which is also tailored for general GAPs with non-unimodular structure, is always the optimal one. The one found by the scheme in [32] is guaranteed to be at most $50 \%$ suboptimal. Moreover, our algorithm allows for directed communication graphs, while the one in [32] assumes undirected communications. Finally, agents in [32] exchange, at each communication round, two real vectors of size $N$ and $M$ respectively and a vector of size $M$ representing which agent is performing each task. As for the distributed approach in [33], each agent has to flood its local variables to all the other agents. This results in multi-hop communications at each iteration. Moreover, the scheme in [33] is based on the assumption of static, undirected graphs. Each agent in [33] sends to its neighbors a real vector of size $M$ and three integers. Similarly to our approach, it considers the solution of a knapsack problem at each iteration. Finally, as [32], it guarantees at most $50 \%$ sub-optimality of the solution found. We perform a comparison between the proposed approach and the one in [33] for the scenario with $N=15, M=30$. The results are in Table III We took for both the schemes the same underlying communication graph. Since the algorithm in [33] needs agents to flood their information to all the other agents at each communication round, we multiply the total number of iterations of the algorithm by $N d$, with $d$ diameter of the graph. Besides the problems generated via Model D, our algorithm is able to find in less iterations a solution with a smaller relative error with respect to the one found by the algorithm in [33].

\section{EXPERIMENTS ON GAPS \\ FOR A TEAM OF GROUND AND AERIAL RoBOTS}

In the following, we provide experimental results on a generalized assignment scenario where a team of heterogeneous (ground and aerial) mobile robots has to accomplish a set of tasks that may not be completely known in advance. We start by describing how we implemented the proposed distributed scheme into the ROS framework. Then, we propose the Distributed Dynamic Assignment and Servicing Strategy, a resolution methodology for this assignment scenario, and provide experiments on a real fleet of ground and aerial robots.

\section{A. Experimental ROS Architecture}

In the proposed architecture, robots are "smart" cyberphysical agents endowed with communication, computation and actuation capabilities. Each cyber-physical agent consists of three ROS nodes, namely Optimization, Planner and Controller ROS nodes, see Figure 3. It is worth noticing that, in general, each agent has a dedicated machine on which these processes run, so that there is no need for a central computing unit handling the agents. The Optimization node handles the steps of the distributed optimization algorithm of the associated cyber-physical agent. It communicates with the Optimization nodes of the other robots through the ROS publisher-subscriber communication protocol according to a fixed communication graph, and exchanges messages containing the local candidate bases. Note that the communication among processes in ROS is completely asynchronous. As shown in the theory this is handled by our distributed algorithm. Each time such process receives a message from a neighbor, a callback function stores the received basis. Each node performs an iteration of the Distributed Branch-and-Price algorithm within a loop of $5 \mathrm{~ms}$. At the beginning of this loop, the node performs one step of the column generation algorithm with the received bases. Then, it sends the updated basis to its neighbors and stays idle until the next loop iteration. The Optimization nodes characterize the Optimization Layer (c.f. Figure 3) of the proposed architecture. The Control and Planner ROS nodes constitute instead the Control Layer of the proposed software. More in detail, the Planner node generates, through polynomial splines, a sufficiently smooth trajectory steering a robot over its designated tasks. The Controller implements a trajectory tracking strategy. It receives the pose of the vehicle by a Vicon motion capture system and sends 
TABLE II

NUMERICAL RESULTS

\begin{tabular}{ccccccc} 
Model & $\mathrm{N}$ & $\mathrm{M}$ & Communication Rounds (Avg-Std) & Relative Error (Avg-Std) & Stored Nodes (Avg-Std) & Time (Avg-Std) \\
\hline & 5 & 20 & $83.30-25.36$ & $0.00 \%-0.00 \%$ & $1.10-0.36$ & $1.94-0.60$ \\
& 5 & 30 & $329.38-151.87$ & $0.01 \%-0.08 \%$ & $1.44-0.64$ & $12.85-5.89$ \\
$\mathrm{~A}$ & 10 & 20 & $75.34-43.86$ & $0.00 \%-0.00 \%$ & $1.30-0.78$ & $3.34-1.86$ \\
& 10 & 30 & $107.92-87.52$ & $0.01 \%-0.07 \%$ & $1.30-1.06$ & $10.29-7.88$ \\
& 15 & 20 & $76.60-26.23$ & $0.01 \%-0.05 \%$ & $1.12-0.38$ & $5.09-2.01$ \\
& 15 & 30 & $95.86-33.27$ & $0.00 \%-0.00 \%$ & $1.08-0.34$ & $14.59-4.80$ \\
\hline & 5 & 20 & $192.04-205.30$ & $1.06 \%-2.10 \%$ & $3.50-3.79$ & $4.67-5.03$ \\
& 5 & 30 & $774.50-680.58$ & $0.57 \%-0.91 \%$ & $5.04-4.40$ & $33.68-29.46$ \\
$\mathrm{~B}$ & 10 & 20 & $161.36-222.29$ & $0.25 \%-0.80 \%$ & $3.14-4.38$ & $6.56-9.13$ \\
& 10 & 30 & $236.36-324.55$ & $0.20 \%-0.53 \%$ & $3.24-4.53$ & $23.42-31.24$ \\
& 15 & 20 & $90.02-42.64$ & $0.02 \%-0.15 \%$ & $1.36-0.66$ & $5.16-2.43$ \\
& 15 & 30 & $178.40-174.70$ & $0.04 \%-0.10 \%$ & $2.16-2.13$ & $27.32-26.81$ \\
\hline & 5 & 20 & $158.24-149.94$ & $0.63 \%-1.25 \%$ & $3.00-3.03$ & $3.95-3.74$ \\
& 5 & 30 & $652.32-741.28$ & $0.48 \%-1.20 \%$ & $4.48-5.59$ & $27.98-31.95$ \\
$\mathrm{C}$ & 10 & 20 & $155.06-230.47$ & $0.47 \%-1.58 \%$ & $3.24-4.93$ & $5.81-8.44$ \\
& 10 & 30 & $375.52-431.19$ & $0.59 \%-1.03 \%$ & $5.50-6.41$ & $35.37-40.32$ \\
& 15 & 20 & $107.08-133.53$ & $0.14 \%-0.57 \%$ & $1.80-2.26$ & $4.85-6.16$ \\
& 15 & 30 & $294.02-306.51$ & $0.24 \%-0.42 \%$ & $3.94-4.15$ & $38.92-39.99$ \\
\hline & 5 & 20 & $1072.76-601.48$ & $4.31 \%-3.88 \%$ & $20.10-11.23$ & $33.47-18.10$ \\
& 5 & 30 & $4805.44-2525.97$ & $4.91 \%-3.65 \%$ & $36.80-18.05$ & $201.56-101.85$ \\
$\mathrm{D}$ & 10 & 20 & $933.76-979.26$ & $2.77 \%-4.12 \%$ & $18.44-20.06$ & $36.20-36.08$ \\
& 10 & 30 & $5959.95-3596.71$ & $4.96 \%-4.26 \%$ & $63.55-38.69$ & $560.14-338.02$ \\
& 15 & 20 & $187.88-164.20$ & $0.22 \%-0.44 \%$ & $2.84-2.52$ & $9.38-8.19$ \\
& 15 & 30 & $6171.65-4015$ & $3.37 \%-2.81 \%$ & $56.15-37.02$ & $740.5-481.8$ \\
\hline
\end{tabular}

TABLE III

PERFORMANCE COMPARISON

\begin{tabular}{ccc|cc|cc}
\hline \multirow{2}{*}{ Model } & \multirow{2}{*}{$\mathrm{M}$} & \multirow{2}{*}{$\mathrm{N}$} & \multicolumn{2}{|c|}{ Distributed Branch-and-Price } & \multicolumn{2}{c}{$[33]$} \\
\cline { 4 - 7 } & & & Comm. Rounds (Avg-Std) & Rel. Error (Avg-Std) & Comm. Rounds (Avg-Std) & Rel. Error (Avg-Std) \\
\hline $\mathrm{A}$ & 15 & 30 & $95.86-33.27$ & $0.00 \%-0.00 \%$ & $411.6-62.44$ & $0.56 \%-0.37 \%$ \\
\hline $\mathrm{B}$ & 15 & 30 & $178.40-174.70$ & $0.04 \%-0.10 \%$ & $447.3-65.77$ & $1.91 \%-1.13 \%$ \\
\hline $\mathrm{C}$ & 15 & 30 & $294.02-306.51$ & $0.24 \%-0.42 \%$ & $453.6-107.9$ & $3.45 \%-1.74 \%$ \\
\hline $\mathrm{D}$ & 15 & 30 & $6171.65-4015$ & $3.37 \%-2.81 \%$ & $302.4-34.12$ & $0.0 \%-0.0 \%$ \\
\hline
\end{tabular}

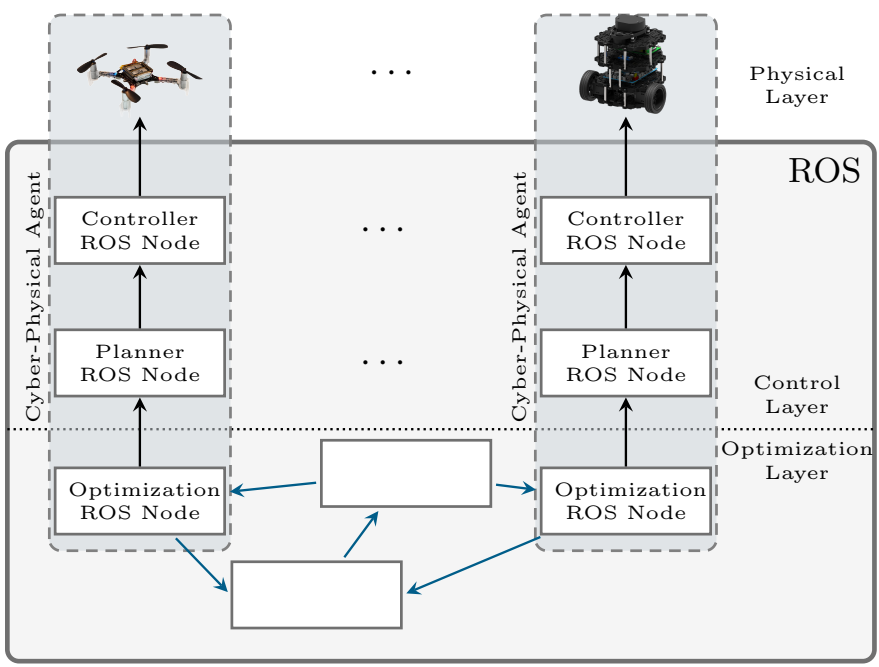

Fig. 3. Distributed Dynamic Assignment and Servicing Strategy architecture. Blue rectangles represent the smart cyber-physical agents endowed with computation, communication and actuation capabilities.

the control inputs to the robot actuators (Physical Layer in Figure 3.

\section{B. Distributed Dynamic Assignemnt: Scenario and Strategy}

The scenario evolves as follows. We consider a team of ground and aerial mobile robots moving in a three-dimensional environment parametrized by a frame $\{x, y, z\}$. A set of tasks, parametrized by a position on the $\{x, y\}$ plane, are scattered in the environment. Some of the tasks can be accomplished only by ground robots, other are accessible only to aerial robots and there are tasks that can be performed by all the robots. For a task to be accomplished, a robot has to visit the task location, stand still for a certain random time $T^{\mathrm{H}}$ and go back to a given depot (e.g., to recharge batteries). As in practical applications, the information about the problem instance is not known in advance and new data arrive while the agents are fulfilling other tasks. To adapt the Distributed Branch-andPrice algorithm to such dynamic scenario, we combine it with the methodology proposed in [26] into an optimization and task-fulfilling approach which we call Distributed Dynamic Assignment and Servicing Strategy. Such procedure combines a distributed optimization phase with a planning and control scheme to steer the robots over the assigned tasks. More in detail, the experiment starts with the cyber-physical agents running the Distributed Branch-and-Price Algorithm on a set of tasks known in advance. Inspired by [32], we pick $p_{i m}$ in (1) as a time-discounted reward, i.e., $p_{i m}=\lambda_{m}^{\tau_{i}^{m}}$ where 
$\lambda_{m} \in(0,1)$ is a scoring value for task $m$ and $\tau_{i}^{m}$ is the time needed by agent $i$ to reach task $m$. The time $\tau_{i}^{m}$ is evaluated as the robot-task distance (on the $\{x, y\}$ plane) scaled by the robot maximum speed $(1 \mathrm{~m} / \mathrm{s}$ for the UAVs and $0.22 \mathrm{~m} / \mathrm{s}$ for the ground vehicles). The fact that a task $m$ is not accessible to a certain robot $i$ is modeled by taking $w_{i m}>g_{i}$ in (1). In the following, we assume that the sets $P_{i}$, generated randomly according to Model A in Section IV, are fixed throughout the scenario evolution. As soon as a robot reaches the designed task, it stands still on the location for a random time $T^{\mathrm{H}}$ between 3 and 5 seconds. In the proposed experiment, we consider a dynamic scenario in which the number of tasks appearing during the evolution is always smaller than the number of served tasks. For the sake of simplicity, we suppose that one new task is made available to robots each time a task has been fulfilled. In this way, the size of the optimization problem is constant. We point out that the strategy can be applied to more general cases where more tasks are revealed. Moreover, while in the current set-up we consider the immediate strategy in which we re-optimize the entire problem, one could think of implementing tailored schemes leveraging the dynamic structure of the problem. As soon as new tasks appear, the cyber-physical agents run the Distributed Branch-and-Price algorithm on a problem including the new tasks and discarding the visited ones. Specifically, the cost vector entries change according to the new task positions. Meanwhile, each robot keeps performing tasks according to its latest allocation. An example of the evolution of this strategy is in Figure 4 A snapshot from an experiment with 2 Crazyflie nano-quadrotors and 3 Turtlebot3 Burger is in Figure 5. Here robots have terminated the distributed optimization procedure and one of the allocations is shown. A video is available as supplementary material to the pape ${ }^{5}$

Remark 5.1: As discussed, e.g., in [3], GAPs can be also used to find approximate solutions of vehicle routing problems (VRPs). In general, VRPs penalize the order of execution of the tasks, and involve a larger number of variables with respect to GAPs. The idea in [3] is to construct a GAP instance based on the VRP problem data. As soon as a GAP solution has been found, robots perform their associated task in an order that minimizes, e.g., the total travelled distance. This can be done, e.g., by solving a Shortest Hamiltonian Path Problem (SHPP). The proposed Distributed Dynamic Assignment and Servicing Strategy could be thus modified in order to address such scenarios. Specifically, robots start solving the GAP with the available tasks and, once an optimal solution has been found, construct robot-to-tasks paths by solving SHPPs. When a new task arrives, robot re-solve the optimization problem and adjust the path according to the new problem data. We performed an experiment with 3 Crazyflie nano-quadrotors and 2 Turtlebot3 Burger with the cloud-based approach. A video is available as supplementary material to the manuscript 6

\footnotetext{
${ }^{5}$ The video is also available at https://youtu.be/S1_3ZmJvvbu

${ }^{6}$ The video is also available at https://youtu.be/vBSJsduFYKQ
}

\section{Conclusion}

In this paper, we proposed a purely distributed branch-andprice approach to solve the Generalized Assignment Problem in a network of agents, endowed with computation and communication capabilities, that are aware of only a small part of the global optimization problem data. Agents cooperatively solve a relaxations of the GAP by means of a distributed column generation algorithm, targeted for this particular scenario involving binary optimization variables. Since the solution of this relaxation may not be feasible for the GAP, agents cooperatively generate and solve new optimization problems, considering each time additional constraints. Finally, we considered an assignment scenario where tasks may appear dynamically during time. We implemented the proposed algorithms in a ROS based testbed and showed results from experiments on a team of ground and aerial vehicles executing the generalized assignment. Future investigations may include the solution of dynamic instances of the GAP with tailored distributed approaches that do not need to re-optimize the entire problem when new data arrive.

\section{ACKNOWLEDGMENT}

The authors would like to thank Alessandro Rucco for the fruitful discussions and Nicola Mimmo for the support during the experiments.

\section{APPENDIX A \\ LINEAR PROGRAMS}

An LP in standard form is a problem in the form

$$
\begin{array}{cl}
\min _{x} & c^{\top} x \\
\text { subj. to } & A x=b, \\
& x \geq 0 .
\end{array}
$$

where $c \in \mathbb{R}^{d}, A \in \mathbb{R}^{r \times d}$ and $b \in \mathbb{R}^{r}$ are the problem data and $x \in \mathbb{R}^{d}$ is the optimization variable. All the problem constraints are expressed as equality constraints and the variables must be non-negative. A column for the problem in (11) is a vector in the form $\left[\begin{array}{cc}c_{\ell} & A_{\ell}^{\top}\end{array}\right] \in \mathbb{R}^{r+1}$ where $A_{\ell}^{\top}$ is the $\ell$-th column of $A$. A basis $B$ is a set of $r$ independent columns of the LP. We denote by $c_{B}\left(A_{B}\right)$ the sub-vector (sub-matrix) of $c(A)$ constructed from the columns in $B$. Assume that a solution $x^{\star}$ to (11) exists. Then, it can be shown that $x^{\star}$ can be decomposed into two sub-vectors $x_{B}^{\star} \neq 0$ of basic variables and $x_{N}^{\star}=0$ of non-basic variables. A basis represents a minimal representation of a linear program, i.e., it is a subset of the problem data representing the problem solution. It can be shown that there exists a basis $B$ such that $x_{B}^{\star}$ is the solution of:

$$
\begin{gathered}
\min _{x} c_{B}^{\top} x \\
\text { subj. to } A_{B} x=b, \\
x \geq 0 .
\end{gathered}
$$




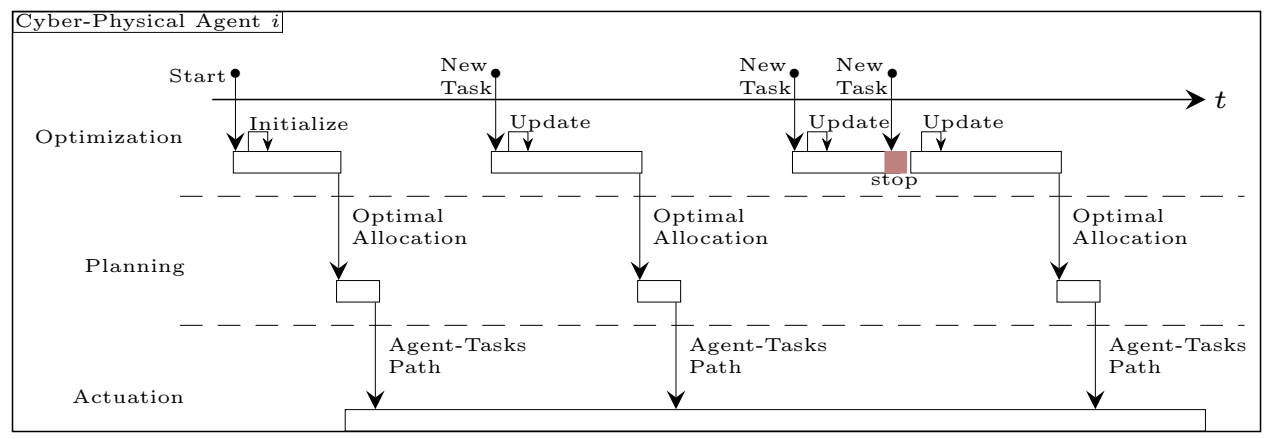

Fig. 4. An example of the Distributed Dynamic Assignment and Servicing Strategy evolution from the perspective of the generic cyber-physical agent. Each time a new task appears, the robot updates the local problem data and re-starts the optimization. If a new task arrives during the re-optimization, this latter is halted (red rectangle) and a new one starts. When robot-to-task paths are evaluated, robot actuation changes accordingly.

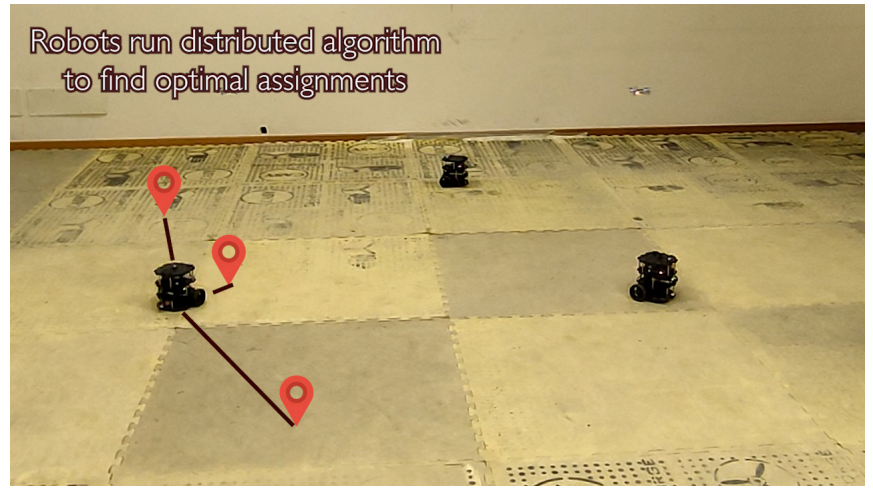

Fig. 5. Snapshot from an experiment. The figure depicts the optimal assignment for one of the robots.

\section{APPENDIX B \\ PROOF OF THEOREM 3.1}

\section{A. Preliminary Lemmas for the Proof of Theorem 3.1}

Before proceeding with the proof of Theorem 3.1. we provide two lemmas which are useful for the analysis.

Lemma B.1: Let Assumption 2.1 hold. Consider a network of $N$ agents running the steps in CASE 1 of Algorithm 1 to solve a node $\mathcal{M \mathcal { P } ^ { \ell }}$ of the tree. Then, in a finite number of iterations, agents reach consensus to an optimal basis $B^{\ell}$ associated with the optimal cost $J^{\star \ell}$ of $\mathcal{M} \mathcal{P}^{\ell}$.

Proof: The proof mimics the one proposed in [23]. We refer the reader to this work for additional details. First, we show that $\mathcal{M P}^{\ell}$ can be obtained by applying the DantzigWolfe decomposition to the following Linear Program:

$$
\begin{aligned}
\max _{z_{1}, \ldots, z_{N}} & \sum_{i=1}^{N} c_{i}^{\top} z_{i} \\
\text { subj. to } & \sum_{i=1}^{N} z_{i}=1_{M}, \\
& z_{i} \in \operatorname{conv}\left(P_{i}^{\ell}\right), i=1, \ldots, N .
\end{aligned}
$$

Indeed, we recall that, for GAPs, the vertexes of $\operatorname{conv}\left(P_{i}^{\ell}\right)$ coincide with the points $v_{i}^{q}, q \in Q_{i}^{\ell}$, [5]. Thus, points $z_{i} \in \operatorname{conv}\left(P_{i}^{\ell}\right)$ can be represented as $z_{i}=\sum_{q=1}^{\left|Q_{i}^{\ell}\right|} v_{i}^{q} \lambda_{i}^{q}$ with $\sum_{q=1}^{\left|Q_{i}^{\ell}\right|} \lambda_{i}^{q}=1$ and $\lambda_{i}^{q} \geq 0$. Let $\Lambda$ be the stack of the variables $\lambda_{i}^{q}$. By substituting these equations in (12) one obtains a problem in the form

$$
\max _{\Lambda} \sum_{i=1}^{N} \sum_{q=1}^{\left|Q_{i}^{\ell}\right|}\left(c_{i}^{\top} v_{i}^{q}\right) \lambda_{i}^{q}
$$

subj. to $\sum_{i=1}^{N} \sum_{q=1}^{\left|Q_{i}^{\ell}\right|} v_{i}^{q} \lambda_{i}^{q}=1_{M}$,

$$
\begin{aligned}
& \sum_{q=1}^{\left|Q_{i}^{\ell}\right|} \lambda_{i}^{q}=1, i=1, \ldots, N, \\
& \lambda_{i}^{q} \geq 0, q \in\left\{1, \ldots,\left|Q_{i}^{\ell}\right|\right\}, i=1, \ldots, N,
\end{aligned}
$$

which is problem $\mathcal{M P}^{\ell}$. Notice that the resulting pricing problem for each agent $i$ is

$$
\begin{gathered}
\max \left(c_{i}-\pi\right)^{\top} z_{i} \\
\text { subj. to } z_{i} \in \operatorname{conv}\left(P_{i}^{\ell}\right) .
\end{gathered}
$$

By definition of convex hull and linearity of the cost function, (13) shares the same optimal vertexes of (7). Thereby, the steps of CASE 1 in Algorithm 1 can be seen as applied to LP (12). At this point, we note that, during the algorithmic evolution, agent $i$ can update its local candidate basis $B_{i}^{t}$ by considering new columns in the local linear program. It is worth noting that, starting from any basis $B_{i}^{t}$, there exists a finite number of pivoting operations to the optimal basis $B^{\ell}$. These columns can be found in two ways: (i) by the local column generation routine and the subsequent pivoting and (ii) when collecting all the in-neighbors matrices $B_{j}^{t}$ with $j \in \mathcal{N}_{i, t}^{\text {in }}$. If $J_{i}^{t}<J^{\star \ell}$, there always exists an agent $j$ able to generate a column improving the cost $J_{i}^{t}$ after a pivoting. Since the network is connected if that column is fundamental for the evolution of the algorithm, e.g., it belongs to the optimal basis, then agent $j$ will generate it (and include it) in its basis within a finite number of communication rounds. Thus, as soon as $J_{i}^{t}<J^{\star \ell}$, there always exists a finite time $T_{D}$ such that $J_{i}^{t}<J_{I}^{t+T_{D}}$. Since there exists only a finite number of columns, in a finite number of communication rounds $T_{f}$ it stands that $J_{i}^{T_{f}}=J^{\star \ell}$ for each $i$. If a lexicographic solver is considered, then it stands that $B_{i}^{t}=B^{\ell}$ for each $i$. This concludes the proof. 
Lemma B.2: Let Assumption 2.1 hold. Then, a processor has computed its final basis and can halt the execution of the steps in CASE 1 of Algorithm 1 as soon as the value of $B_{i}^{t}$ has not changed after $L(2 N-1)$ communication rounds.

Proof: Assume that a certain node $i$ satisfies $B_{i}^{t}=$ $B^{\star}, J_{i}^{t}=J^{\star}$ for all $t \in\left\{t_{0}, \ldots, t_{0}+2 L(N-1)\right\}$, and pick any other node $j$. Without loss of generality, consider $t_{0}=0$. By $L$-strong connectivity, after at most $L$ communication rounds, agent $i$ has been able to spread its basis at least to another agent. We now define the set $\overline{\mathcal{N}}_{0}$ of agents $k \in\{1, \ldots N\}$ such that there exists an increasing sequence of time instants $\tau_{0}, \ldots, \tau_{m}$ comprised between 0 and $L$ (i.e., with $0 \leq \tau_{0}$ and $\left.\tau_{m} \leq L\right)$, such that the edges $\left(i, \ell_{1}\right), \ldots,\left(\ell_{m}, k\right)$ belong to the digraph at times $\tau_{0}, \ldots, \tau_{m}$. This set is not empty, since the union graph is strongly connected in $[0, L]$. Then it stands $J_{k}^{L} \geq J_{i}^{t}, \forall k \in \overline{\mathcal{N}}_{0}$. Consider now the interval $[L, 2 L]$, for which we define a set similar to $\overline{\mathcal{N}}_{0}$, but with paths originating from the agents in $\overline{\mathcal{N}}_{0} \cup\{i\}$. Formally, consider the set $\overline{\mathcal{N}}_{1}$ of agents $k \in\{1, \ldots N\}$ such that there exists an increasing sequence of time instants $\tau_{0}, \ldots, \tau_{m}$ comprised between $L$ and $2 L$ (i.e., with $L \leq \tau_{0}$ and $\tau_{m} \leq 2 L$ ), such that the edges $\left(h, \ell_{1}\right), \ldots,\left(\ell_{m}, k\right)$ belong to the digraph at times $\tau_{0}, \ldots, \tau_{m}$, for some $h \in \overline{\mathcal{N}}_{0} \cup\{i\}$. Notice that $\overline{\mathcal{N}}_{0} \subset \overline{\mathcal{N}}_{1}$, so that $\overline{\mathcal{N}}_{1}$ has a larger cardinality than $\overline{\mathcal{N}}_{0}$. Otherwise, the graph would not be strongly connected in $[L, 2 L]$. Then it stands $J_{k}^{2 L} \geq J_{i}^{t}$, $\forall k \in \overline{\mathcal{N}}_{1}$. Iterating at most $N-1$ times, we see that the sets $\overline{\mathcal{N}}_{0} \ldots, \overline{\mathcal{N}}_{N-2}$ become larger and larger, so that $j \in \overline{\mathcal{N}}_{N-2}$. Thus, it stands that $J_{k}^{(N-1) L} \geq J_{i}^{t}$. That is, after $(N-1) L$ communication rounds, all the agents have at least the same cost agent $i$ had at time 0 . By repeating the same arguments for the converse path, we conclude that $J_{i}^{2(N-1) L} \geq J_{k}^{(N-1) L}$. But, by assumption, $J_{i}^{2(N-1) L}=J^{\star}$, so that we conclude $J^{\star} \leq J_{j}^{(N-1) L} \leq J^{\star}$, i.e., $J_{j}^{(N-1) L}=J^{\star}$. Thus, if $B_{i}^{t}$ does not change for $L(2 N-1)$ time instants, then its value will never change afterwards because all bases $B_{j}^{t}, j \in\{1, \ldots, N\}$, have cost equal to $J^{\star}$ at least as early as time equals $L N$.

\section{B. Proof of Theorem 3.1}

In order to prove the statement, we show that there exists a monotonically increasing time sequence $\left\{\bar{t}_{\ell}\right\}_{\ell \in\left\{0, \ldots, \ell_{\text {end }}\right\}}$, for some $\ell_{\text {end }} \in \mathbb{N}$, such that, at each $\bar{t}_{\ell}$ :

i) For all $i, j \in\{1, \ldots, N\}, \mathcal{T}_{i}^{\bar{t}_{\ell}}=\mathcal{T}_{j}^{\bar{t}_{\ell}}, \mathcal{M P}_{i}^{\ell}=$ $\mathcal{M P}_{j}^{\ell}=\mathcal{M} \mathcal{P}^{\ell}, \mathcal{L}_{i}^{\bar{t}_{\ell}}=\mathcal{L}_{j}^{\bar{t}_{\ell}}=\ell$ and there exists some $i \in\{1, \ldots, N\}$ such that $\mathcal{L}_{i}^{\bar{t}_{\ell}-1} \neq \ell$;

ii) in a finite number of communication rounds, at a time $\bar{t}_{\ell+1} \leq \bar{t}_{\ell}+Q^{\ell}, Q^{\ell} \in \mathbb{N}$, either $\mathcal{M P}_{i}^{\ell+1}=\mathcal{M} \mathcal{P}_{j}^{\ell+1}$ (with $\mathcal{T}_{i}^{\bar{t}_{\ell+1}}=\mathcal{T}_{j}^{\bar{t}_{\ell+1}}$ and $\left.\mathcal{L}_{i}^{\bar{t}_{\ell+1}}=\mathcal{L}_{j}^{\bar{t}_{\ell+1}}, \forall i, j \in\{1, \ldots, N\}\right)$ or agents halt the distributed algorithm, i.e., $\ell=\ell_{\text {end }}$, with $J_{i}^{\bar{t}_{\ell+1}}=J^{\star}$ and $z_{[i]}^{\bar{t}_{\ell+1}}=z^{\star}$ optimal cost and solution of (2) for all $i \in\{1, \ldots, N\}$.

First notice that i) holds trivially at $t_{0}=0$, since all the agents start solving the relaxed version of (5), namely $\mathcal{M} \mathcal{P}^{0}$, and each agent initializes $\mathcal{L}_{i}^{0}=0$. Now, we assume that i) holds for some $\ell$ and prove that ii) holds. Then, by applying the arguments in Lemma B.1 agents reach consensus, in a finite number of communication rounds $\bar{Q}^{\ell}$, on a basis $B^{\ell}$ corresponding to an optimal solution $\Lambda^{\star \ell}$ of $\mathcal{M} \mathcal{P}^{\ell}$. Moreover, by Lemma B.2 each agent $i$ can halt, at some time $\bar{Q}^{\ell} \leq t_{i, \ell} \leq \bar{t}_{\ell}+\bar{Q}^{\ell}+2 L N+1$, the steps of CASE 1 if its basis $B_{i}^{t}$ has not changed for $2 L N+1$ communication rounds (c.f. Algorithm 1). At these times, each agent obtains the same cost $J^{\star \ell}$ and solution $z^{\star \ell}$ of $\mathcal{M P} \mathcal{P}^{\ell}$ (retrieved from $\Lambda^{\star \ell}$ by applying (3)) and sets $\mathcal{L}_{i}^{t_{i, \ell}+1}=\ell+1$. If CASE 2.1 in Algorithm 1 occurs, then each agent $i \in\{1, \ldots, N\}$ sets $J_{i}^{t_{i, \ell}+1}=J^{\star \ell}$ and $z_{[i]}^{t_{i, \ell}+1}=z^{\star \ell}$. Instead, if CASE 2.2 occurs, each agent expands the local tree $\mathcal{T}_{i}^{t_{i, \ell}+1}$. Notice that agents run the BRANCH routine on the same data $\left(\mathcal{T}_{i}^{\bar{t}_{\ell}}\right.$ and $z^{\star \ell}$ ), so they update the same tree with the same new problems. Finally, if there are still problems to be solved in $\mathcal{T}_{i}^{t_{i, \ell}+1}$, each agent extracts a new problem $\mathcal{M} \mathcal{P}_{i}^{\ell+1}$. Since the routine EXTRACTCONSTR is common to all the agents and the constructed trees are identical, $\mathcal{M P}_{i}^{\ell+1}=\mathcal{M P}^{\ell+1}$ for all $i$. Otherwise, if $\mathcal{T}_{i}^{t_{i, \ell+1}}$ is empty, each agent halts the Distributed Branch-and-Price Algorithm. Let $Q^{\ell}=\bar{Q}^{\ell}+2 L N+2$ and let $\bar{t}_{\ell+1}=\max _{i}\left\{t_{i, \ell}\right\}+1$. From the above arguments, $\bar{t}_{\ell} \leq \bar{t}_{\ell+1} \leq \bar{t}_{\ell}+Q^{\ell}$.

Now we show that, if agents halt the distributed algorithm, i.e., $\ell=\ell_{\text {end }}$, then $J_{i}^{\bar{t}_{\ell+1}}=J^{\star}$ and $z_{[i]}^{\bar{t}_{\ell+1}}=z^{\star}$ for all $i \in$ $\{1, \ldots, N\}$, with $J^{\star}$ and $z^{\star}$ optimal cost and solution of (2). First, notice that $J_{i}^{\bar{t}_{\ell+1}}$ and $z_{[i]}^{t_{\ell+1}}$ are the optimal cost value and solution of some problem $\mathcal{M} \mathcal{P}^{\ell}$ such that $J^{\star \ell} \geq J_{i}^{t}$ for each $t \leq \bar{t}_{\ell+1}$ and $z^{\star \ell} \in\{0,1\}^{N M}$. Since (2) is feasible, and in the branch-and-price algorithm all the nodes of the tree are explored (except the ones discarded during the pruning operation) then each agent has run at least one time the steps in CASE 2.1. Thereby, $J_{i}^{\bar{t}_{\ell+1}}$ must be equal to the optimal cost value $J^{\star}$ of (2) and, similarly, $z_{[i]}^{\bar{t}_{\ell+1}}=z^{\star}$ optimal solution to (2). To conclude, we underline that agents can generate only a finite number of problems. Indeed, the number of additional constraints $\left(z_{i_{k}}=0\right.$ and $\left.z_{i_{k}}=1\right)$ they can add is at most $2^{N M}$. Thus, there exists a time $\bar{T}=\bar{t}^{\ell_{\text {end }}}+Q^{\ell_{\text {end }}}$ in which all the agents must halt the distributed scheme. This concludes the proof.

\section{REFERENCES}

[1] T. Öncan, "A survey of the generalized assignment problem and its applications," INFOR: Information Systems and Operational Research, vol. 45, no. 3, pp. 123-141, 2007.

[2] M. Savelsbergh, "A branch-and-price algorithm for the generalized assignment problem," Oper. research, vol. 45, no. 6, pp. 831-841, 1997.

[3] M. L. Fisher and R. Jaikumar, "A generalized assignment heuristic for vehicle routing," Networks, vol. 11, no. 2, pp. 109-124, 1981.

[4] S. Martello and P. Toth, "Generalized assignment problems," in Intern. Symp. on Algorithms and Computation. Springer, 1992, pp. 351-369.

[5] C. Barnhart, E. L. Johnson, G. L. Nemhauser, M. W. Savelsbergh, and P. H. Vance, "Branch-and-price: Column generation for solving huge integer programs," Oper. research, vol. 46, no. 3, pp. 316-329, 1998.

[6] B. P. Gerkey and M. J. Matarić, "A formal analysis and taxonomy of task allocation in multi-robot systems," The International Journal of Robotics Research, vol. 23, no. 9, pp. 939-954, 2004.

[7] E. Hartuv, N. Agmon, and S. Kraus, "Scheduling spare drones for persistent task performance under energy constraints," in Proc. of the 17th Intern. Conf. on Auton. Agents and MultiAgent Systems. Intern. Found. for Auton. Agents and Multiagent Systems, 2018, pp. 532-540.

[8] J. Bellingham, M. Tillerson, A. Richards, and J. P. How, "Multi-task allocation and path planning for cooperating UAVs," in Cooperative control: models, applications and algo. Springer, 2003, pp. 23-41. 
[9] M. C. Gombolay, R. J. Wilcox, and J. A. Shah, "Fast scheduling of robot teams performing tasks with temporospatial constraints," IEEE Transactions on Robotics, vol. 34, no. 1, pp. 220-239, 2018.

[10] M. Turpin, N. Michael, and V. Kumar, "An approximation algorithm for time optimal multi-robot routing," in Algorithmic Foundations of Robotics XI. Springer, 2015, pp. 627-640.

[11] T. Shima, S. Rasmussen, and D. Gross, "Assigning micro UAVs to task tours in an urban terrain," IEEE Transactions on Control Systems Technology, vol. 15, no. 4, pp. 601-612, 2007.

[12] D. P. Bertsekas, "The auction algorithm: A distributed relaxation method for the assignment problem," Annals of operations research, vol. 14, no. 1 , pp. $105-123,1988$.

[13] M. B. Dias, B. Kannan, B. Browning, E. Jones, B. Argall, M. F. Dias, M. Zinck, M. Veloso, and A. Stentz, "Sliding autonomy for peer-to-peer human-robot teams," in Proceedings of the international conference on intelligent autonomous systems, 2008, pp. 332-341.

[14] D. A. Castanón and C. Wu, "Distributed algorithms for dynamic reassignment," in IEEE Conference on Decision and Control (CDC), vol. 1, 2003, pp. 13-18.

[15] K. Lerman, C. Jones, A. Galstyan, and M. J. Matarić, "Analysis of dynamic task allocation in multi-robot systems," The International Journal of Robotics Research, vol. 25, no. 3, pp. 225-241, 2006.

[16] M. Alighanbari and J. P. How, "Decentralized task assignment for unmanned aerial vehicles," in IEEE Conference on Decision and Control $(C D C), 2005$, pp. 5668-5673.

[17] C. Nam and D. A. Shell, "Robots in the huddle: Upfront computation to reduce global communication at run time in multirobot task allocation," IEEE Transactions on Robotics, 2019.

[18] M. Hassan, D. Liu, S. Huang, and G. Dissanayake, "Task oriented area partitioning and allocation for optimal operation of multiple industrial robots in unstructured environments," in 13th Intern. Conf. on Control Autom. Robotics \& Vision (ICARCV). IEEE, 2014, pp. 1184-1189.

[19] N. Karapetyan, J. Moulton, J. S. Lewis, A. Q. Li, J. M. O'Kane, and I. Rekleitis, "Multi-robot dubins coverage with autonomous surface vehicles," in 2018 IEEE International Conference on Robotics and Automation (ICRA). IEEE, 2018, pp. 2373-2379.

[20] S. Chopra, G. Notarstefano, M. Rice, and M. Egerstedt, "A distributed version of the hungarian method for multirobot assignment," IEEE Transactions on Robotics, vol. 33, no. 4, pp. 932-947, 2017.

[21] M. Bürger, G. Notarstefano, F. Bullo, and F. Allgöwer, "A distributed simplex algorithm for degenerate linear programs and multi-agent assignments," Automatica, vol. 48, no. 9, pp. 2298-2304, 2012.

[22] A. Settimi and L. Pallottino, "A subgradient based algorithm for distributed task assignment for heterogeneous mobile robots," in IEEE Conference on Decision and Control (CDC), 2013, pp. 3665-3670.

[23] M. Bürger, G. Notarstefano, and F. Allgöwer, "Locally constrained decision making via two-stage distributed simplex," in IEEE Conferece on Decision and Control and European Control Conference (CDCECC), 2011, pp. 5911-5916.

[24] E. Montijano, D. Tardioli, and A. R. Mosteo, "Distributed dynamic sensor assignment of multiple mobile targets," in 2019 IEEE/RSJ International Conference on Intelligent Robots and Systems (IROS). IEEE, 2019, pp. 4921-4926.

[25] A. R. Mosteo, E. Montijano, and D. Tardioli, "Optimal role and position assignment in multi-robot freely reachable formations," Automatica, vol. 81, pp. 305-313, 2017.

[26] S. Karaman and G. Inalhan, "Large-scale task/target assignment for UAV fleets using a distributed branch and price optimization scheme," IFAC Proceedings Volumes, vol. 41, no. 2, pp. 13 310-13 317, 2008.

[27] V. Pilloni, M. Franceschelli, L. Atzori, and A. Giua, "Deployment of applications in wireless sensor networks: a gossip-based lifetime maximization approach," IEEE Transactions on Control Systems Technology, vol. 24, no. 5, pp. 1828-1836, 2016.

[28] A. Kwok and S. Martinez, "A distributed deterministic annealing algorithm for limited-range sensor coverage," IEEE Transactions on Control Systems Technology, vol. 19, no. 4, pp. 792-804, 2011.

[29] L. Abbatecola, M. P. Fanti, G. Pedroncelli, and W. Ukovich, "A distributed cluster-based approach for pick-up services," IEEE Trans. on Automation Science and Engin., vol. 16, no. 2, pp. 960-971, 2018.

[30] A. Testa, A. Rucco, and G. Notarstefano, "A finite-time cutting plane algorithm for distributed mixed integer linear programming," in Conference on Decision and Control (CDC). IEEE, 2017, pp. 3847-3852.

[31] _ - "Distributed mixed-integer linear programming via cut generation and constraint exchange," IEEE Transactions on Automatic Control, vol. 65 , no. 4 , pp. 1456-1467, 2019.
[32] H.-L. Choi, L. Brunet, and J. P. How, "Consensus-based decentralized auctions for robust task allocation," IEEE Transactions on Robotics, vol. 25, no. 4, pp. 912-926, 2009.

[33] L. Luo, N. Chakraborty, and K. Sycara, "Distributed algorithm design for multi-robot generalized task assignment problem," in IEEE/RSJ Intern. Conf. on Intelligent Robots and Systems. IEEE, 2013, pp. 4765-4771.

[34] _ "Distributed algorithms for multirobot task assignment with task deadline constraints," IEEE Transactions on Automation Science and Engineering, vol. 12, no. 3, pp. 876-888, 2015.

[35] R. K. Williams, A. Gasparri, and G. Ulivi, "Decentralized matroid optimization for topology constraints in multi-robot allocation problems," in 2017 IEEE International Conference on Robotics and Automation (ICRA). IEEE, 2017, pp. 293-300.

[36] N. Buckman, H.-L. Choi, and J. P. How, "Partial replanning for decentralized dynamic task allocation," in AIAA Scit. Forum, 2019, p. 0915.

[37] Z. Talebpour and A. Martinoli, "Adaptive risk-based replanning for human-aware multi-robot task allocation with local perception," IEEE Robotics and Automation Letters, vol. 4, no. 4, pp. 3790-3797, 2019.

[38] F. Vanderbeck, "On Dantzig-Wolfe decomposition in integer programming and ways to perform branching in a branch-and-price algorithm," Operations Research, vol. 48, no. 1, pp. 111-128, 2000.

[39] P. C. Gilmore and R. E. Gomory, "A linear programming approach to the cutting-stock problem," Op. res., vol. 9, no. 6, pp. 849-859, 1961.

[40] S. Martello, "Knapsack problems: algorithms and computer implementations," Wiley-Interscience series in discrete math. and optim., 1990.

[41] C. N. Jones, E. C. Kerrigan, and J. M. Maciejowski, "Lexicographic perturbation for multiparametric linear programming with applications to control," Automatica, vol. 43, no. 10, pp. 1808-1816, 2007.

[42] F. Farina, A. Camisa, A. Testa, I. Notarnicola, and G. Notarstefano, "Disropt: a python framework for distributed optimization," IFACPapersOnLine, vol. 53, no. 2, pp. 2666-2671, 2020. 